\title{
EL PARQUE DE DIVERSIONES COMO LABORATORIO DE FÍSICA MECÁNICA.
}

\author{
AMUSEMENT PARK \\ AS A MECHANICS PHYSICS LABORATORY \\ Xiomara del Pilar Murillo Castañeda ${ }^{1}$ \\ Jaime Duvan Reyes ${ }^{2}$
}

\begin{abstract}
Resumen
El presente trabajo muestra los resultados obtenidos en el aprovechamiento de las instalaciones de un parque de diversiones para la apropiación de conceptos físicos tales como; movimiento circular, trabajo y energía, entre otros, vistos como una práctica de laboratorio desde la misma experiencia. Para esto se diseñó y aplicó un material guía; usado como medio de aprendizaje, que relaciono la teoría, la experiencia y las temáticas de estudio. La experiencia se desarrolló con un grupo de estudiantes de de física de mecánica uno de la universidad distrital Francisco José de Caldas de Bogotá-Colombia, en sesiones tanto en el aula de clase como en el parque de diversiones y fue evaluada en tres momentos (antes, durante y después de la visita al parque). Los análisis de resultados y conclusiones muestran el nivel de desarrollo en las competencias, conceptos, dimensiones del conocimiento y fortalezas del proceso, evidenciando un avance en la significación y construcción de los elementos tratados
\end{abstract}

Palabras Clave: Parque de diversiones, Practica de laboratorio, módulos de enseñanza, construcción, significación y aprendizaje

\begin{abstract}
Abstrac
This paper shows the results of the use of the premises of an amusement park for the appropriation of concepts such as physical, circular motion, work and energy, among others, seen as a lab from the same experience. For this reason we designed and implemented a guidance material, used as a means of learning, which relate the theory, experience and topics of study. The experiment was carried out with a group of students from a mechanical physics from the University Francisco José de Caldas District of Bogota, Colombia, in sessions both in the classroom and in the amusement park and was assessed three times (before during and after the visit to the park). The analysis of results and conclusions show the level of development in the skills, concepts, dimensions of knowledge and strengths of the process, showing an improvement in the significance and construction of the items discussed
\end{abstract}

Keywords: Amusement, laboratory, teaching modules, construction, meaning

\footnotetext{
${ }^{1}$ Universidad Distrital Francisco José de Caldas. Xpmurillo375@gmail.com

${ }^{2}$ Universidad Distrital Francisco José de Caldas,,dreyesr@udistrital.edu.co
} 


\section{Introducción}

Al ingresar a un parque de diversiones, la idea que se viene a la cabeza es que es un lugar de esparcimiento, sin tener en cuenta la posibilidad de aprendizaje que este puede tener para la enseñanza de la física, ya que el diseño y funcionamiento de sus atracciones están dados bajo los principios de esta ciencia y su utilización permitiría investigar y servir de objeto de aprendizaje para algunos conceptos de la mecánica clásica.

De esta manera, la visita y utilización de un parque de diversiones, puede ser medio de enseñanza si se tiene un objetivo claro donde se relacionen la teoría y la experiencia de los objetos de aprendizaje que los estudiantes han de alcanzar a construir. Es por ello que el presente trabajo está dirigido a docentes y estudiantes de manera que puedan tener pautas y orientaciones para la visita y el mejor aprovechamiento de los recursos que el parque de diversiones proporciona de manera que las atracciones y su uso se conviertan en una metodología de aprendizaje para la enseñanza de la física.

\section{Metodología}

En esta etapa se presenta el diseño y aplicación del material guía para la visita al parque de diversiones de dos atracciones, tales como; Montaña Rusa y centrox, dividido en tres etapas. La primera de éstas (previa a la visita al parque) consistió en una fase introductoria y de sensibilización que comprende el objetivo de la práctica, el despliegue de un dispositivo de motivación hacia el aprendizaje y para indagar por los preconceptos del tema. También contempla el nivel exigido para el desarrollo de habilidades en el cálculo, para la experiencia y su posterior análisis, así como para la descripción de los fenómenos físicos en sus respectivas variables de medición.

En la segunda etapa se presenta una guía estructurada a partir de preguntas para consignar las observaciones; en este espacio se desarrolla la toma de medidas y tratamiento de problemas que tienen que ver con las temáticas a tratar de cada atracción. Se pretende que el estudiante corrobore sus ideas previas a partir de la experiencia, tome medidas, desarrolle preguntas de tipo analítico consignadas en una guía y tome todo el conjunto de lo anterior como una práctica de laboratorio. Se espera que la información que recibe a partir de la experiencia, con el conocimiento previo que tiene el estudiante le permita reestructurar sus conceptos y apropiarse de ellos de manera significativa para un mejor aprendizaje. Este modulo se desarrolla en las instalaciones del parque de diversiones.

En la tercera etapa de los módulos se proporciona una guía al estudiante para que presente un informe escrito y sustentado de los problemas, las medidas, las relaciones entre las diferentes variables que ha tomado en el momento anterior. 
$5^{\circ}$ CONGRESO NACIONAL DE ENSEÑANZA DE LA FÍSICA.

Universidad Pedagógica Nacional (U. P. N)

Universidad Distrital Francisco José de Caldas (U. D. F. J. C)

Bogotá, Colombia. 16 al 20 de mayo 2011

Un grupo de estudiantes del curso de Mecánica Clásica de la

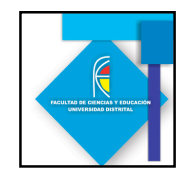

Licenciatura en física de la Universidad Distrital Francisco José de Caldas fue para tal fin. Para la elección del perfil de este grupo, se tuvo en cuenta que contaran con los

prerrequisitos conceptuales de las temáticas que se relacionan en cada atracción seleccionada.

La información recolectada es de forma magnética (audio y video) y escrita, tomada a partir de los módulos de aprendizaje, esta fue sometida a los métodos estadísticos más apropiados, tales como media, varianza, entre otros de tal forma que sean razonables entre las diferentes variables encontradas.

\section{Resultados}

\section{Variables en el desarrollo experimental}

A partir del diseño y preparación de las actividades implementadas para la recolección de datos se establecen las siguientes variables de estudio.

\section{Resultados de los niveles de competencia}

Se analizo el proceso de desarrollo inicial y final de habilidades competitivas, tales como; interpretativa, propositiva y argumentativa, para los módulos propuestos en la Montaña rusa y Centrox.

De los datos recolectados en la prueba diagnostico inicial se observa un promedio del $70 \%$, en el nivel de competencia de los estudiantes. Indicando en un inicio que estos se encuentran en un nivel aceptable. La competencia con mayor dominio inicial es la interpretativa con $75 \%$, le sigue la propositiva con $71.6 \%$ y por último se encuentra la argumentativa con $66 \%$.

Posteriormente al desarrollo del material didáctico hecho en el parque, los estudiantes realizan un informe de laboratorio, desde el trabajo en el aula de clase hasta la salida de campo. A partir de allí se observo que la competencia con mayor dominio al finalizar la salida de campo es la propositiva, le sigue la interpretativa y en última escala continúa siendo la argumentativa.

El promedio es del $90 \%$ en el nivel de competencias que se desarrolla en el ejercicio de la montaña rusa aumentando en un $20 \%$ en relación a la prueba diagnóstico inicial. De lo anterior se puede asegurar que al final los estudiantes se encuentran ubicados en un estatus sobresaliente para esta actvidad.

Para el ejercicio del Centrox el promedio es de $90 \%$ en el nivel de competencias, aumentando en un $20 \%$ en relación a la prueba diagnostico inicial. Lo que indica que al finalizar los estudiantes obtuvieron una mejoría en sus desempeños.

Resultados de Preguntas de forma individual 
$5^{\circ}$ CONGRESO NACIONAL DE ENSEÑANZA DE LA FÍSICA.

Universidad Pedagógica Nacional (U. P. N)

Universidad Distrital Francisco José de Caldas (U. D. F. J. C)

Bogotá, Colombia. 16 al 20 de mayo 2011

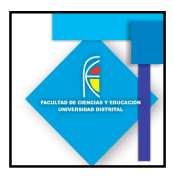

Se analizo el proceso de desarrollo inicial y final de la apropiación de conceptos físicos indicando el número de respuestas acertadas en la prueba diagnostico inicial y final. Esto con la finalidad de comparar dichos resultados.

Se observo un incremento en cada estudiante en las pruebas de salida comparadas con las pruebas de entrada. La media de la prueba diagnostico es $\bar{x}=3.65$ y la desviación estándar está dada por $\sigma=1.2020$ de lo que se puede deducir que la calificación más baja de la prueba inicial está dada en 2.5 y la más alta en 4.5 .

La prueba de salida de la montaña rusa tiene una media de $\bar{x}=4.155$ aumentando en un $10 \%$ en relación a la prueba diagnostico. La desviación estándar es de $\sigma=1.13$, por lo tanto la calificación más alta de la prueba de salida es de 5.0 y la más baja está dada en 3.055

Para la prueba de salida del Centrox el promedio esta dado por $\bar{x}=4.44$

aumentando en $20 \%$ en relación a la prueba diagnostico. La desviación estándar es de $\sigma=0,5$. Siendo el resultado de esta prueba superior en relación a la Montaña Rusa; destacando que cada estudiante obtuvo una puntuación mayor de alrededor de una unidad en las dos pruebas de salida en comparación con la prueba diagnostico.

\section{Discusión}

1. Aprovechamiento del Parque de Diversiones: el estudio realizado demuestra que hay situaciones de motivación que proporcionan condiciones que facilitan el aprendizaje, como es el caso de la práctica de laboratorio en el parque de diversiones. Este contexto permite a los estudiantes desarrollar y mejorar sus niveles de competencia y en particular la competencia propositiva ya que la evaluación en procesos asociados a la aplicación de conceptos, la creación, el diseño mostró un desarrollo favorable de pensamiento convergente y divergente.

2. La Planeación de la visita al parque: se evidencia la importancia de un objetivo claro expuesto en los módulos de trabajo teniendo en cuenta el dispositivo de motivación en los estudiantes y un deseo por entender el funcionamiento de las atracciones para así poder explicar cada una de las sensaciones, lo que facilita la recolección de datos. Las actitudes observadas permiten comprobar la importancia de diseñar una estrategia didáctica acorde con las atracciones de un parque de diversiones para así poder desarrollar el estimulo en los estudiantes. Así mismo se observa el valor de la práctica de campo como estrategia didáctica, ya que permite una apropiación en la comprensión de los fenómenos, pues en la experiencia se presentan sensaciones que abren camino al descubrimiento y a una significación personal del conocimiento.

3. La importancia de la motivación: los estudiantes que asistieron al parque de diversiones manifestaron motivación en cada una de las actividades, esto se muestra en la actitud y dinamismo que presentaron en el transcurso de todo el proceso. Para ello se tiene como evidencia los comentarios hechos en 
$5^{\circ}$ CONGRESO NACIONAL DE ENSEÑANZA DE LA FÍSICA.

Universidad Pedagógica Nacional (U. P. N)

Universidad Distrital Francisco José de Caldas (U. D. F. J. C)

Bogotá, Colombia. 16 al 20 de mayo 2011

grabaciones (ver anexo $N^{0} 1$ ) en donde se manifiesta una completa satisfacción en la metodología de enseñanza.

4. El contexto de aprendizaje: de acuerdo con el desarrollo de este proyecto una situación de aprendizaje en mecánica puede caracterizarse por la observación de algunos aspectos importantes, tales como:

4.1 La existencia de comunicación entre el estudiante y sus compañeros.

4.2 Elementos de conexión entre el conocimiento previo y la experiencia para construir los conceptos;

4.3 El desarrollo de situaciones contextuales (reales) en donde se evidencie la posibilidad de construir conceptos asociados a los fenómenos de la mecánica clásica, este caso los relacionaos con las atracciones del parque de diversiones,

4.4 La necesidad de buscar respuestas a cada una de las conexiones entre la teoría física y las sensaciones, esto revela el papel del docente y la necesidad de una preparación previa a la práctica de cada uno de los temas específicos que se consideran importantes en el desarrollo de habilidades en los niveles de competencia.

\section{Referencias Bibliográficas}

[1] Alvares A, Rincón. C, Suarez. H, Segura. D, Tamayo. F, Torres. N, Boada. M \&Quijano. A. (2006). Grandes iconos de la ciudad: El navegador pedagógico.(pp. 206). Secretaria de educación. Bogotá, Colombia.

[2] Einstein. A \& Infeld. L. (1986). "La evolución de la física", (1ra Ed.).. Salvat Editores S.A., Barcelona.

[3] Conceição Oliveira M. Visita monitoreada a un museo de ciencias ies posible aprender? Tesios de doctorado Universidad de são paulo. são paulo Brasil.

[4] Hewitt. P. (1999), Física Conceptual, (3ra Ed.), City college of San Francisco, California,Person.

[5] Robert L. Wolke, 2007 ., Lo que Einstein le contó a su barbero (1a Ed.),Barcelona España

[6] Serway R. A y Jewett. J.W (2005). Física para la ciencia e ingeniería Tomo. I. (6 $\left.6^{\mathrm{a}} \mathrm{Ed}\right)$ International Thomson editores, S.A.

[7] Thiagarajan S y Parker G., (2000), Equipos de trabajo actividades y juegos de integración, (Edición en español), México.: Pretice Hall.

[8] Tipler P.A y Mosca. G (2005). Física para la ciencia y la tecnología. (5a Ed). Barcelona, España. :Editorial Reverte S.A. 
$5^{\circ}$ CONGRESO NACIONAL DE ENSEÑANZA DE LA FÍSICA.

Universidad Pedagógica Nacional (U. P. N)

Universidad Distrital Francisco José de Caldas (U. D. F. J. C)

Bogotá, Colombia. 16 al 20 de mayo 2011 